\title{
Why Most Indian Young Women are Unhappy with Their Sex Lives
}

\author{
Hajare $\mathbf{R}^{*}$ \\ Indian Council of Medical Research, National AIDS Research Institute, India
}

*Corresponding author: Rahul Hajare, Post Doc Indian Council of Medical Research, National AIDS Research Institute, New Deli, India, Tel: 9921707584; Email: rahulhajare17@gmail.com

\section{Case Report}

Volume 3 Issue 2

Received Date: April 02, 2020

Published Date: April 30, 2020

DOI: $10.23880 /$ abca-16000117

\section{Abstract}

Women who habitually monitored their appearance, and for whom appearance determined their level of physical self-worth experienced lower sexual satisfaction. Skin in the game and one out of three Indian women cheat on their spouses. Skin pulp is saying you have single centric sex or multiple sex partners. Cope with multiple sex partners. Purity is a measure of the amount of potency present in a relation compared to those of related people, impure, high side women

Keywords: Female Sexual Dysfunction; Ethical Philosophy; Relating to the law; Sex lives

\section{Introduction}

A new research suggests that most women remain unhappy and stressed about their sex lives with at least one in five of them suffering from female sexual dysfunction. The study has conducted by the researchers of Pune University and according to the study, half of young Indian women experience sexually-related personal distress, with one in five women having at least one female sexual dysfunction (FSD).The study conducted by the Women's Health Research Program at Pune University has reported, for the first time, an overall picture of the sexual wellbeing of Indian women between the ages of 18 and 39.Results showed 50.2 percent of young Indian women experienced some form of sexuallyrelated personal distress. This relates to the degree of feeling guilty, embarrassed, stressed or unhappy about their sex lives [1]. A concerning 29.6 percent of women experienced sexually-related personal distress without dysfunction, and 20.6 per cent had at least one FSD. The most common FSD was low sexual self-image, which caused distress for 11 percent of study participants. Arousal, desire, orgasm and responsiveness dysfunction affected 9 percent, 8 percent, 7.9 percent and 3.4 percent of the study cohort respectively. Sexual self-image dysfunction has associated with being overweight, obese, living together with partner, not married, married and breastfeeding. Taking psychotropic medication (such as antidepressants), reported by 20 percent of surveyed women, had the most pervasive impact on sexual function. The use of the combined oral contraceptive pill has not associated with any sexual dysfunction. Sexual wellbeing is recognised as a fundamental human right. It is of great concern that one in five young women has an apparent sexual dysfunction and half of all women within this age group experience sexually-related personal distress [2]. This is a wake-up call to the community and signals the importance of health professionals being open and adequately prepared to discuss young women's sexual health concerns, recruited 6986 women aged 18 to 39 years, living in Pune, Maharashtra and Aurangabad, to take partin the study. As a part of the study, all women completed a questionnaire that assessed their sexual wellbeing in terms of desire, arousal, responsiveness, orgasm, and self-image. Participants also evaluated whether they had sexually-associated personal distress and provided extensive demographic information. Almost one-third of participants described themselves as single, 47percent had a body mass index within the normal range, and nearly 70 percent had reported being sexually active in the 30 days preceding the study. Women who habitually monitored their appearance, and for whom appearance determined their level of physical self-worth, reported being less sexually assertive and more self-conscious during intimacy and experienced lower sexual satisfaction $[3,4]$. If untreated, sexually-related personal distress and FSD could impact relationships and overall quality of life as women aged. The high prevalence of sexually-related personal distress signals the importance of health professionals, particularly those working in the fields of gynaecology and fertility, being adequately prepared to routinely ask young women about any sexual 
health concerns, and to have an appropriate management or referral pathway in place. While the medical community cannot vouch for the exact mechanism, experts do know that the fluctuation of one's hormone levels before and during the orgasm can impact how the breasts feel during that time. More specifically the not increasing levels of estrogen in the first two weeks of romance can cause your breasts to get bigger, while increasing amounts of progesterone during the second half may puff up milk ducts [5]. The combination of this can lead to breasts pain and feeling tender. Since every woman is different, her symptoms and orgasm will also be different. But some things will always be in common, some of these things that your breasts may face during sexual act. Here are a few steps you can take to curb changes in bored sex life cycle: Eat a diet lower in fat, avoiding high-fat foods. Skip caffeine, which means no coffee, tea, cola, and chocolate. Avoid the salt for a couple of weeks before your period starts. Wear a bra that fits you properly and provides good support. Aim for a daily cardio workout. Word to the wise, if dealing with sexual pleasure-related soreness, still bring it up with doctor if it's bothering sexual life. Together, can figure out the best way and find relief.

\section{Conclusion}

The researchers concluded that the findings have important implications for policy and prevention and should inform the creation of more effective sexual health education programs and interventions. The research has an observational study, so no firm conclusions could be drawn about the cause and effect. The authors pointed to several limitations including that it did not involve women who have sex with men unhappy0, or any other gender/sexuality minority groups, which could limit the generalisation of the findings. Researcher also noted that a hypothetical scenario may not invoke the same emotional response or reflect reallife behaviour.

\section{Acknowledgment}

I acknowledge for this important work with Honorable Respected Dr. R.S.Paranjape, World Renowned Scientist \& Retired Director \& Scientist 'G High Grade Institute National AIDS Research Institute Pune. This has inspired \& captured the imagination and attention of across the research and pure service.

\section{References}

1. Rahul H (2019) Non-Medical Basis Characterization of Orgasm Associated with Approach Sex Can Last up to 20 Seconds to 15 Minutes, Eligible Women Individuals' Poor Transportation Facility of Private Pharmacy Institutions in Pune, India. Adv Neur Neur Sci 2(1): 1-2.

2. Rahul H (2018) Why Men are Missing from Fertility Debates. J Gastrointest Disord Liver Func 4(2): 38-39.

3. Rahul H (2019) Focus on right kissing short line to long line a retrospective very rare lesion for both Indian males and females: Results of a classical laboratory R \& D study. Journal of Neurology \& Neurophysiology 10: 6.

4. Rahul H (2019) An Important Study for Care Perspective Tops Self-Reported Private Co- Educational By Pass Pharmaceutical Institutions in Number of Abandoned Children. Advances in Bioengineering \& Biomedical Science Research 2(4): 1-2.

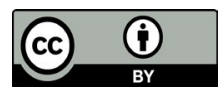

